International Journal of Modern Physics D

(C) World Scientific Publishing Company

\title{
A THEORETICAL CASE FOR NEGATIVE MASS-SQUARE FOR SUB-eV PARTICLES*
}

\author{
D. V. AHLUWALIA-KHALILOVA ${ }^{\dagger}$ \\ Center for Studies of Physical, Mathematical, and Biological Structure of Universe \\ Department of Mathematics, Ap. Postal C-600 \\ University of Zacatecas (UAZ), Zacatecas, ZAC 98062, Mexico \\ IRINA DYMNIKOVA \\ Department of Mathematics and Computer Science, University of Warmia and Mazury \\ Żotnierska 14, Olsztyn 10-561, Poland
}

\begin{abstract}
Electroweak gauge bosons have masses of the order of $10^{2} \mathrm{GeV}\left[/ \mathrm{c}^{2}\right]$, while masses of additional bosons involved in gravito-electroweak unification are expected to be still higher. These are at least eleven orders of magnitude higher than sub-eV range indications for neutrino masses. Under these circumstances we suspect that the sub-eV particles are created in a spacetime where gravitational effects of massive gauge bosons may become important. The question that we thus ask is: What is the spacetime group around a gravito-electroweak vertex? Modeling it as de-Sitter we find that sub-eV particles may carry a negative mass square of the order of $-\left(3 / 8 \pi^{3}\right)\left(M_{\text {unif. }} / M_{\text {Planck }}\right)^{4} M_{\text {Planck }}^{2}$. Neutrino oscillation data then hints at $30-75 \mathrm{TeV}$ scale for $M_{\text {unif., }}$ where $M_{\text {unif. }}$ characterizes gravito-electroweak unification scale.
\end{abstract}

\section{Introduction}

One of the lessons from E. P. Wigner's early work $\$$ is that the notion of mass is not an arbitrary physical construct but takes its origin from constancy of speed of light for all inertial observers. The latter implies description of physical states in terms of the Casimir invariants associated with the Poincaré group:

$$
C_{1}=P_{\mu} P^{\mu}, \quad C_{2}=W_{\mu} W^{\mu},
$$

with Pauli-Lubanski pseudovector, $W_{\mu}$, defined as

$$
W_{\mu}=-\frac{1}{2} \epsilon_{\mu \nu \rho \sigma} J^{\nu \rho} P^{\sigma} .
$$

Here we use the notation of Ref. 2]. Each representation space is then characterized by eigenvalues of these Casimir operators. Representation spaces of the type $(j, 0) \oplus$ $(0, j)$ are characterized by a

*This essay received an "honorable mention" in the 2003 Essay Competition of the Gravity Research Foundation.

${ }^{\dagger}$ E-mail: d.v.ahluwalia-khalilova@heritage.reduaz.mx

‡E-mail: irina@matman.uwm.edu.pl 
(a) Positive definite mass, and

(b) Single spin- $j$

while spaces of the type $[(j, 0) \oplus(0, j)](1 / 2,1 / 2)$ carry interpretation of 35

(i) Positive definite mass, but

(ii) Indefinite/multiple spin.

Attempts to force a single-spin interpretation - as for Rarita-Schwinger framework - result in well-known problems $\underline{4}$. Efforts to implement a single-spin interpretation on $[(j, 0) \oplus(0, j)](1 / 2,1 / 2)$ spaces are akin to insisting on a "particle" interpretation for the Dirac's $(1 / 2,0) \oplus(0,1 / 2)$ representation space by imposing a covariant constraint which throws away the "negative energy" sector, i.e., the antiparticles. Or, at least this is a view we have put forward in Refs. 3, 5].

Now, with the discovery of massive gauge bosons of electroweak interactions a new situation has arisen. This, as we shall now argue, may question positive/real definiteness of mass for sub-eV particles.

The massive gauge bosons have masses of the order of $10^{2} \mathrm{GeV}\left[/ c^{2}\right]$. These are at least eleven orders of magnitude higher than sub-eV range indication for neutrino masses 6 -17. Our thesis arises from the possibility that the sub-eV particles are created in a spacetime where gravitational effects of massive gauge bosons may become important.

The question that we thus ask is: What is the spacetime group around a gravitoelectroweak vertex? In the context of Refs. 18, 19, if we impose the requirements of (a) spherical symmetry, (b) dominant energy condition for a source term, (c) regularity of density, and (d) finiteness of mass; then, the answer is de SitterSchwarzschild geometry. Thus, in the interaction region the spacetime symmetry group is de Sitter. This may be taken as our fundamental working assumption. ${ }^{\text {a }}$

For the de Sitter-Schwarzschild case, the stress-energy tensor evolves smoothly from de Sitter vacuum $T_{\mu \nu}=\rho_{0} c^{2} g_{\mu \nu}$ at $r=0$ to Minkowski vacuum $T_{\mu \nu}=0$ at infinity. Here $\rho_{0}$ is the mass density at the origin, and shall be identified with the gravito-electroweak scale. The induced metric is given by 20 .

$$
d s^{2}=\left(1-\frac{2 G M(r)}{c^{2} r}\right) c^{2} d t^{2}-\left(1-\frac{2 G M(r)}{c^{2} r}\right)^{-1} d r^{2}-r^{2} d \theta^{2}-r^{2} \sin ^{2} \theta d \phi^{2},
$$

with

$$
M(r)=4 \pi \int_{0}^{r} \rho(x) x^{2} d x
$$

\footnotetext{
a This approximation neglects spin of the massive gauge bosons which may, when properly ac-
} counted for, may be responsible for explaining neutrino mixing matrix. 
For any density profile satisfying the requirements (b)-(d) enumerated above, asymptotic behavior in the $r \rightarrow 0$ region is dictated by (b) and is de Sitter vacuum:

$$
d s^{2}=\left(1-\frac{r^{2}}{r_{0}^{2}}\right) c^{2} d t^{2}-\left(1-\frac{r^{2}}{r_{0}^{2}}\right)^{-1} d r^{2}-r^{2} d \theta^{2}-r^{2} \sin ^{2} \theta d \phi^{2},
$$

with

$$
r_{0}^{2}=\frac{3 c^{2}}{8 \pi G \rho_{0}} .
$$

For $r \rightarrow \infty$, the asymptotic is Schwarzschild

$$
d s^{2}=\left(1-\frac{2 G m}{c^{2} r}\right) c^{2} d t^{2}-\left(1-\frac{2 G m}{c^{2} r}\right)^{-1} d r^{2}-r^{2} d \theta^{2}-r^{2} \sin ^{2} \theta d \phi^{2},
$$

where $m=M(r \rightarrow \infty)$ is the ADM mass.

\section{Negative mass-square for sub-eV particles}

We now envisage that a sub-eV particle, which we may think of as neutrino to be concrete, is created in the de Sitter region and later propagates to $r \rightarrow \infty$ (i.e., to $\mathrm{r} \gg r_{0}$ region) where spacetime is Minkowskian to a good approximation. In the creation region, such a particle is characterized as an eigenstate of the de Sitter Casimir invariants, $\left|I_{1}^{\prime}, I_{2}^{\prime}\right\rangle$. The $I_{1}^{\prime}, I_{2}^{\prime}$ are eigenvalues, respectively, of the de Sitter Casimir operators 21 , b

$$
I_{1}=\Pi_{\mu} \Pi^{\mu}-\frac{1}{2 r_{0}^{2}} J_{\mu \nu} J^{\mu \nu},
$$

and $I_{2}$ (see, Ref. [21, for its definition). In going from Poincaré to de Sitter symmetries, the notion of mass must undergo an unavoidable change. To investigate this modification, we shall concentrate on $I_{1}$ only. The $\Pi_{\mu}$ is defined as,

$$
\Pi_{\mu}=\left(1+\frac{r^{2}-c^{2} t^{2}}{4 r_{0}^{2}}\right) P_{\mu}+\frac{1}{2 r_{0}^{2}} x^{\nu} J_{\mu \nu} .
$$

In the interaction region $r^{2}-c^{2} t^{2} \ll r_{0}^{2}$. ${ }^{\mathrm{c}}$ So, $I_{1}$ approximates to:

$$
I_{1} \approx P_{\mu} P^{\mu}-\frac{1}{r_{0}^{2}}\left(\mathbf{J}^{2}-\mathbf{K}^{2}\right) .
$$

Or, equivalently

$$
I_{1} \approx C_{1}-\frac{1}{r_{0}^{2}}\left(\mathbf{J}^{2}-\mathbf{K}^{2}\right)
$$

where, we remind, $C_{1}$ is the first Casimir operator of the Poincaré group. Eigenvalue of $C_{1}$, up to a multiplicative factor of $c^{2}$, is identified with square of the mass of the particle, $\mu^{2}$.

${ }^{\mathrm{b}}$ Note that $\eta_{\mu \nu}$ of Ref. 21 and that used here differ by a minus sign.
${ }^{\mathrm{c}}$ It shall be confirmed explicitly below towards the end of next section. 
In order to study implications for sub-eV neutrinos, we now evaluate the dragged $I_{1}$ for $(1 / 2,0)$ and $(0,1 / 2)$ representations spaces. ${ }^{\mathrm{d}}$ The right handed and left handed fields inhabit these spaces, respectively. For the $(1 / 2,0)$ representation space, we have 2 ,

$$
\mathbf{J}=\hbar \frac{\boldsymbol{\sigma}}{2}, \quad \mathbf{K}=-i \hbar \frac{\boldsymbol{\sigma}}{2}
$$

while for the $(0,1 / 2)$ representation space,

$$
\mathbf{J}=\hbar \frac{\boldsymbol{\sigma}}{2}, \quad \mathbf{K}=+i \hbar \frac{\boldsymbol{\sigma}}{2} .
$$

This, immediately yields:

$$
I_{1} \approx P_{\mu} P^{\mu}-\frac{\hbar^{2}}{2 r_{0}^{2}} \sigma^{2}
$$

Its eigenvalues are: ${ }^{\mathrm{e}}$

$$
I_{1}^{\prime}=\mu^{2} c^{2}-\frac{3 \hbar^{2}}{2 r_{0}^{2}}
$$

It is now explicit that the notion of mass is modified in going form one spacetime symmetry group to another. As we shall shortly see, this modification allows for negative mas-square for sub-eV particles if gravito-electroweak unification occurs at $\mathrm{TeV}$ scales.

This is the central result of this essay and may offer a natural explanation for certain anomalous results which have come to be known as "negative mass squared problem" for $\bar{\nu}_{e} 23-27$, and $\nu_{\mu} 28$ even though, as outlined in the Addenda, efforts in data analysis tend toward imposing by hand the requirement of physical $m_{\nu}^{2}>0$.

Thus the negative mass-squared values for sub-eV particles, and neutrinos in particular, may be expected to be governed by parameter:

$$
m_{\text {neg. }}^{2}=-\frac{3 \hbar^{2}}{2 r_{0}^{2} c^{2}}
$$

\section{Hint for a $\mathrm{TeV}$ scale gravito-electroweak unification}

If $\rho_{0}$ is identified with an (yet unknown) electroweak-gravitation mass scale $M_{\text {unif. }}$ then, on recalling the definition of $r_{0}$ from Eq. (6), we have

$$
m_{\text {neg. }}^{2}=-\frac{4 \pi G \hbar^{2}}{c^{4}} \rho_{0}
$$

${ }^{\mathrm{d}}$ See, Ref. [5], for the definition of a dragged Casimir. Also, recall that, $J_{i j}=-J_{j i}=\epsilon_{i j k} J_{k}$ and $J_{i 0}=-J_{0 i}=-K_{i}$, with each of the $i, j, k$ taking the values $1,2,3$. The $\mathbf{J}$ are then generators of Lorentz rotations and $\mathbf{K}$ are generators of Lorentz boosts.

e Note, it corrects Ref. 22]. 


$$
\begin{aligned}
& =-\frac{4 \pi G \hbar^{2}}{c^{4}}\left(M_{\text {unif. }} /\left[\left(\frac{4 \pi}{3}\right)\left(\frac{2 \pi \hbar}{M_{\text {unif. }}}\right)^{3}\right]\right), \\
& =-\frac{3}{8 \pi^{3}} \frac{G}{\hbar c} M_{\text {unif. }}^{4}
\end{aligned}
$$

Identifying, $\sqrt{\hbar c / G}$ with $M_{\text {Planck }}$ the above expression becomes,

$$
m_{\text {neg. }}^{2}=-\frac{3}{8 \pi^{3}}\left(\frac{M_{\text {unif. }}}{M_{\text {Planck }}}\right)^{4} M_{\text {Planck }}^{2}
$$

If $M_{\text {unif. }}$ is set to be $100 \mathrm{GeV}$, i.e. of the order of masses for electroweak gauge bosons $W^{ \pm}$and $Z$, one immediately sees that $m_{\text {neg. }}^{2} \simeq-8.4 \times 10^{-15} \mathrm{eV}^{2}$. Existing data on neutrino masses rules out this identification because it is natural to expect that $m_{\text {neq. }}^{2} \sim-\Delta m^{2}$. Where, $\Delta m^{2}$ as derived from atmospheric and solar neutrino data is [36. $\Delta m_{A T M}^{2}=2.5 \times 10^{-3} \mathrm{eV}^{2}\left[/ c^{4}\right], \Delta m_{S O L}^{2}=6.9 \times 10^{-5} \mathrm{eV}^{2}\left[/ c^{4}\right]$.

However, $\left(M_{\text {unif. }} / M_{\text {Planck }}\right)^{4}$-sensitivity of $m_{\text {neg. }}^{2}$ suggests a TeV scale for $M_{\text {unif. }}$. This can be seen explicitly by setting $m_{\text {neg. }}^{2} \sim-\Delta m^{2}$. Then $M_{\text {unif. }}$ reads:

$$
M_{\text {unif. }} \sim\left(\frac{8 \pi^{3}}{3} \frac{\Delta m^{2}}{M_{\text {Planck }}^{2}}\right)^{1 / 4} M_{\text {Planck }}
$$

The atmospheric neutrino data implies a $M_{\text {unif. }} \simeq 74 \mathrm{TeV}$ while the cited solar neutrino mass-squared difference yields, $M_{\text {unif. }} \simeq 30 \mathrm{TeV}$. These $M_{\text {unif. }}$, correspond, respectively, to $r_{0} \simeq 5 \times 10^{-4} \mathrm{~cm}$, and $r_{0} \simeq 3 \times 10^{-3} \mathrm{~cm}$.

\section{Conclusion}

In view of these considerations, and additional and earlier work of Simicevic 37 , it appears that to discard experimentally indicated $m_{\nu}^{2}<0$ for electron and muon neutrinos may be unwise. The best route may be to look at the data and experiments afresh and allow that it may indeed be that

$$
m_{\nu_{e}}^{2}<0, \quad m_{\nu_{\mu}}^{2}<0
$$

At the same time a global analysis of data on neutrinos - specifically data on neutrino oscillations, data on neutrino-less double beta decay, data on the end point of tritium beta decay, and $\pi^{+} \rightarrow \mu^{+}+\nu_{\mu}-$ must be done to allow for $m_{\nu}^{2}<0$. If negative mass-square is finally established for a sub-eV particle it would be necessary to device experiments which may distinguish between various proposals 24 which suggest, or attempt to accommodate, negative mass squares.

\section{Addenda}

We think that the following additional information may be helpful to the reader of our essay:

$\underline{\text { (Anti)Electron neutrino mass }}$

The latest publication on $m_{\nu_{e}}^{2}$ at the time of sending this essay to IJMPD seems 
to be from Lobashev 38 . It gives $m_{\mu_{e}}^{2}=-2.5 \pm 2.5 \pm 2.0 \mathrm{eV}^{2}$. Particle data group 39 , gives: $m_{\nu_{e}}^{2}=-2.5 \pm 3.3 \mathrm{eV}^{2}$ and only includes results of Lobashev 40 and Weinheimer $\underline{41}$ with the following two observations:

(1) The data were corrected for electron trapping effects in the source, eliminating the dependence of the fitted neutrino mass on the fit interval. The analysis assuming a pure beta spectrum yields significantly negative fitted $m_{\nu}^{2} \approx-(20-10) \mathrm{eV}^{2}$. This problem is attributed to a discrete spectral anomaly of about $6 \times 10^{-11}$ intensity with a time-dependent energy of $5-15 \mathrm{eV}$ below the endpoint. The data analysis accounts for this anomaly by introducing two extra phenomenological fit parameters resulting in a best fit of $m_{\nu}^{2}=$ $-1.9 \pm 3.4 \pm 2.2 \mathrm{eV}^{2}$ which is used to derive a neutrino mass limit. However, the introduction of phenomenological fit parameters which are correlated with the derived $m_{\nu}^{2}$ limit makes unambiguous interpretation of this result difficult.

(2) We do not use the following data for averages, fits, limits, etc. ... Stoeff 23 . $m_{\nu}^{2}=-130 \pm 20 \pm 15 \mathrm{eV}^{2} \ldots$ Robertson $\frac{42}{}: m_{\nu}^{2}=-147 \pm 68 \pm 41 \mathrm{eV}^{2}$.

In order to put all these matters in perspective one of us has taken liberty of asking one of the early experimentalists about his views on his own experiment. His candid reply reads,

We still have no clue why there was an excess of counts close to the betaendpoint in our spectra. The experiment is "mothballed" since 1993 and has not been operated since. We were not able to come up with a satisfactory explanation for the bump at the end of the spectrum. In the meantime, The neutrino group in ... improved their spectrometers. They had initially similar puzzling result, but in the meantime, the neutrino mass extracted from their spectra is consistent with zero or a very small value.

and further strengthens the need for careful experiments without any prejudice in data analysis for $m_{\nu}^{2}>0$.

$\underline{\text { Muon neutrino mass }}$

Additional hint for negative mass-square for neutrino masses resides in two possible values - determined by which value of pion mass one uses - for $m_{\nu_{\mu}}^{2} \cdot \underline{39}$.

$$
\begin{aligned}
& \text { Solution B: } \quad-0.016 \pm 0.023 \mathrm{MeV}^{2} \text {, } \\
& \text { Solution A: } \quad-0.143 \pm 0.024 \mathrm{MeV}^{2} \text {. }
\end{aligned}
$$

${ }^{\mathrm{f}}$ Taking $m_{\text {neg. }}^{2} \sim-10^{2} \mathrm{eV}^{2}$ yields $M_{\text {unif }} \sim 10^{3} \mathrm{TeV}$.

${ }^{\mathrm{g}}$ Taking $m_{\text {neg. }}^{2} \simeq-0.143 \mathrm{MeV}^{2}$ yields $M_{\text {unif }} \simeq 2.0 \times 10^{5} \mathrm{TeV}$. 


\section{Acknowledgments}

DVA-K thanks colleagues in neutrino community for clarification on the status of experiments on beta-endpoint spectra and Majorana/Dirac nature of neutrinos, Irina Dymnikova for her warm hospitality in Olsztyn, a referee for his helpful remarks, and N. Simicevic for bringing his work on the subject to our attention. This published version was duly updated after we learned of Simicevic's work.

This work was supported by the Polish Committee for Scientific Research through the grant 5P03D.007.20. Additional support was provided by CONACyT Project 32067-E.

\section{References}

1. E. P. Wigner, "On unitary representations of the inhomogeneous Lorentz group," Ann. Math., 40, 149-204 (1939).

2. L. H. Ryder, Quantum field theory, (Cambridge University Press, Cambridge, 1987).

3. D. V. Ahluwalia, M. Kirchbach, " $(1 / 2,1 / 2)$ Representation space: An ab initio construct," Mod. Phys. Lett. A, 16, 1377-1384 (2001).

4. A. E. Kaloshin, V. P. Lomov "Propagator of the interacting Rarita-Schwinger field," hep-ph/0307272, and references therein.

5. M. Kirchbach, D. V. Ahluwalia, "Spacetime structure of massive gravitino," Phys. Lett. $B$, 529, 124-131 (2002).

6. K. Lande et al., "Solar neutrino observations with Homestake Cl-37 detector," AIP Conf. Proc. 243, 1122-1133 (1992).

7. R. Davis, "Solar neutrinos. II: Experimental," Phys. Rev. Lett., 12, 303-305 (1964).

8. J. N. Bahcall, "Solar neutrinos before and after KAMLAND," JHEP, 0302, 009 (2003).

9. M. B. Smy (for the Super-Kamiokande Collaboration), "Five Years of Neutrino Physics with Super-Kamiokande," Proceedings of the XXth International Conference on Neutrino Physics and Astrophysics (in press),, hep-ex/0206016

10. , Q. R. Ahman et al. (SNO collaboration), "Direct evidence for neutrino flavor transformation from neutral current interactions in the Sudbury neutrino observatory," Phys. Rev. Lett., 89, 011301 (2002).

11. , K. Eguchi et al. (KamLAND collaboration), "First results from KamLAND: Evidence for reactor anti-neutrino disappearance," Phys. Rev. Lett., 90, 021802 (2003).

12. A. Bazarko, for the BooNE Collaboration, "Status of MiniBooNE," Proceedings of the 31st International Conference on High Energy Physics (ICHEP02) Amsterdam, 24-31 July 2002 (in press), hep-ex/0210020

13. H. V. Klapdor-Kleingrothaus, A. Dietz, H. L. Harney, I. V. Krivosheina, "Evidence for neutrinoless double beta decay," Mod. Phys. Lett. A, 16, 2409-2420 (2001).

14. H. V. Klapdor-Kleingrothaus, A. Dietz, I. V. Krivosheina, "Status of evidence for neutrinoless double beta decay," Found. Phys., 32, 1181-1223 (2002).

15. H. V. Klapdor-Kleingrothaus, "One year of evidence of neutrinoless double beta decay," hep-ph/0302237

16. C. Athanassopouloset al. (LSND Collaboration), Evidence for $\bar{\nu}_{\mu} \rightarrow \bar{\nu}_{e}$ oscillations from the LSND experiment at LAMPF, Phys. Rev. Lett., 77, 3082-3085 (1996).

17. C. Athanassopoulos et al. (LSND Collaboration), "Evidence for $\nu_{\mu} \rightarrow \nu_{e}$ oscillations from LSND," Phys. Rev. Lett., 81, 1744-177 (1998).

18. I. Dymnikova, "The cosmological term as a source of mass," Class. Quant. Grav.," 19, 725-737, (2002). 
19. I. Dymnikova, "Spherically symmetric space-time with the regular de Sitter center," Int. J. Mod. Phys. D, 12, 1015-1034 (2003).

20. I. Dymnikova, "Vacuum nonsingular black hole," Gen. Rel. Grav., 24, 235-242 (1992).

21. F. Gürsey, "In, Group Theoretical concepts and methods in elementary particle physics: Lectures of the Istanbul Summer School in Theoretical Physics, Ed. F. Gürsey, (Gordon and Breach, New York, 1964).

22. D. V. Ahluwalia-Khalilova, I. Dymnikova, "Spacetime as origin of neutrino oscillations," hep-ph/0305158 Version 1.

23. W. Stoeffl, D. J. Decman, "Anomalous structure in the beta decay of gaseous molecular tritium," Phys. Rev. Lett., 75, 3237-3240 (1995).

24. G. J. Stephenson, Jr., T. Goldman, "A possible solution to the tritium endpoint problem," Phys. Lett. B, 440, 89-93 (1998).

25. J. Ciborowski, J. Rembielinski, "Tritium decay and the hypothesis of tachyonic neutrinos," Eur. Phys. J. C, 8, 157-161 (1999).

26. R. Ehrlich, "Implications for the cosmic ray spectrum of a negative electron neutrino mass squared," Phys. Rev. D, 60, 017302 (1999).

27. R. Ehrlich, "Neutrino mass squared inferred from the cosmic ray spectrum and tritium beta decay," journal="Phys. lett. B", 493, 229-232 (2000).

28. C. Caso et al. (Particle Data Group), Euro. Phys. J C, 3, 1- (1998).

29. K. Assamagan et al., Phys. Lett. B, 335, 231- (1994).

30. K. Assamagan et al., Phys. Rev D, 53, 6065- (1996).

31. O. Jinnouchi, K. Homma, "Can negative mass square of the electron neutrino be an indication of degenerated relic neutrinos," Phys. Lett. B, 435, 381-388, (1998).

32. M. Dine, L. Randall, S. Thomas, "Baryogenesis from flat directions of the supersymmetric standard model," Nucl. Phys. B, 458, 291-326 (1999).

33. R. N. Mohapatra, S. Nussinov, "Can long range anomalous neutrino interactions account for the measured tritium beta decay spectrum," Phys. Lett. B, 395, 63-68 (1997).

34. J. I. Collar, "Endpoint structure in beta decay from coherent weak-interaction of the neutrino," hep-ph/9611420

35. T. Chang, G. Ni, "Explanation of negative mass-square of neutrinos," hep-ph/0009291

36. S. Pakvasa, J. W. F. Valle, "Neutrino properties before and after KAMLAND," hep-ph/0301061

37. N. Simicevic, "Implications of weak-interaction space deformation for neutrino mass measurements," Int. J. Mod. Phys. D (this issue), nucl-th/9909034

38. V. M. Lobashev, "The search for the neutrino mass by direct method in the tritium beta-decay and perspectives of study it in the project KATRIN," Nucl. Phys. A, 719, 153c-160c (2003).

39. K. Hagiwara et al. (Particle Data Group), Phys. Rev. D, 66, 010001 (2002).

40. V. M. Lobashev et al., "Direct search for mass of neutrino and anomaly in the tritium beta spectrum," Phys. Lett. B, 460, 227-235 (1999).

41. C. Weinheimer, "High precision measurement of the tritium $\beta$ spectra near its end point and upper limit on the neutrino mass," Phys. Lett. B, 460, 219- (1999).

42. R. G. H. Robertson et al., "Limits on $\bar{\nu}_{e}$ mass from observations of the $\beta$ decay of molecular tritium," Phys. Rev. Lett., 67, 957- (1991). 